\title{
Hidden Order in the Cuprates
}

\author{
Sudip Chakravarty ${ }^{1}$, R. B. Laughlin ${ }^{2}$, Dirk K. Morr ${ }^{3}$, Chetan Nayak ${ }^{1}$ \\ 1 Physics Department, University of California Los Angeles, Los Angeles, CA 90095-1547 \\ ${ }^{2}$ Department of Physics, Stanford University, Stanford, CA 94305 \\ 3 Theoretical Division, Los Alamos National Laboratory, Los Alamos, NM 87545
}

(October 24, 2018)

\begin{abstract}
We propose that the enigmatic pseudogap phase of cuprate superconductors is characterized by a hidden broken symmetry of $d_{x^{2}-y^{2}}$-type. The transition to this state is rounded by disorder, but in the limit that the disorder is made sufficiently small, the pseudogap crossover should reveal itself to be such a transition. The ordered state breaks time-reversal, translational, and rotational symmetries, but it is invariant under the combination of any two. We discuss these ideas in the context of ten specific experimental properties of the cuprates, and make several predictions, including the existence of an as-yet undetected metal-metal transition under the superconducting dome.

PACS numbers: 71.10.Hf, 71.27.+a, 74.72.-h, 71.10.Pm
\end{abstract}

\section{INTRODUCTION}

In this paper we argue that much of the strange phenomenology of the cuprate superconductors may be simply explained as the disorder-frustrated development of a new order parameter. There are a number of potential candidates for this order, but the one we favor on phenomenological grounds is orbital antiferromagnetism [1] or $d$-density wave (DDW) order [2.3], which is characterized by a local order parameter which distills the universal physics underlying the staggered flux state [4] divorced from the uncontrolled approximations associated with the gauge theory formalism. The essence of our idea is that the pseudogap [9] observed in underdoped cuprates is an actual gap in the one-particle excitation spectrum at the wavevector $(\pi, 0)$ and symmetry-related points of the Brillouin zone associated with the development of this new order. It is "pseudo" in experiment only because of extreme sensitivity to sample imperfection caused by proximity to the phase transition. Moreover, the DDW couples weakly to common experimental probes, and is thus difficult to detect.

Our proposal has much in common with theoretical ideas already in the literature [8 19], and borrows heavily from them. For example, Wen and Lee have proposed staggered currents that fluctuate but do not order [8]. Varma has proposed currents which alternate in the unit cell but do not break translational symmetry [10]. Emery and Kivelson [11.12] and Caprara et al. [13] have proposed states with broken symmetries of different kinds. Our strategy for constructing a theory and confronting experiments differs from most others in deemphasizing modeling of the "strange metal" behavior and focusing on order, low-temperature phenomenology, and material imperfection - all issues with sharp experimental dichotomies amenable to falsification. DDW order can be detected if it is present. If it is not present, the proposal is disproved.
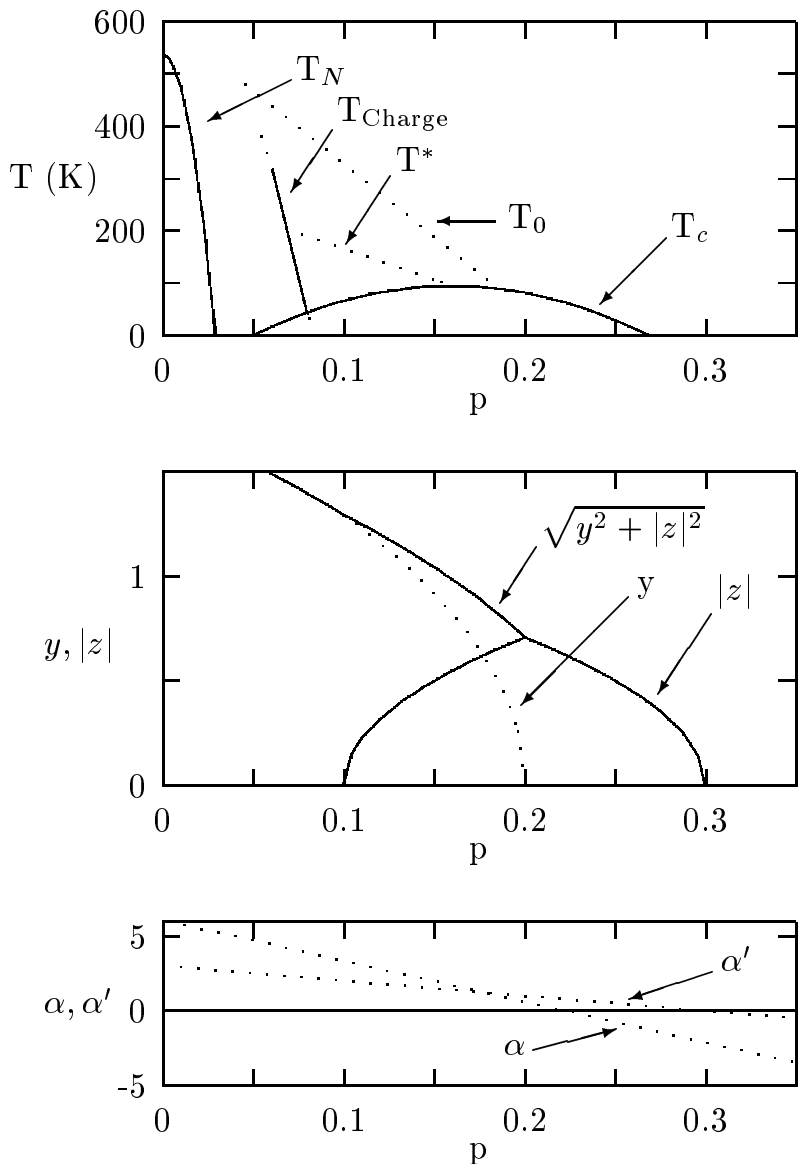

FIG. 1. Top: Phase diagram constructed from experiments on $\mathrm{YBa}_{2} \mathrm{Cu}_{3} \mathrm{O}_{7-\delta}$. $T_{N}$ is the Néel transition, $T_{c}$ is the superconducting transition, $T^{*}$ is the pseudogap crossover [27], $\mathrm{T}_{0}$ is the location of the maximum in the uniform susceptibilty, and $T_{\text {Charge }}$ is a charge-ordering line recently reported by Mook et al. [39]. Middle: Values of $|z|$ (solid), y (dots) and $\left(y^{2}+|z|^{2}\right)^{1 / 2}$ (solid) minimizing the free energy of Eq. (1) with $\lambda=1, \gamma=-0.8$, and the linear functions $\alpha$ and $\alpha^{\prime}$ shown on the bottom. The parameter $p$ is hole doping. 

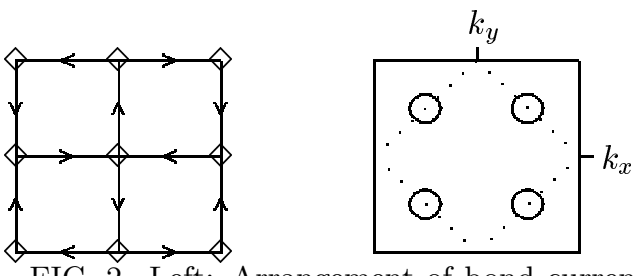

FIG. 2. Left: Arrangement of bond currents in the DDW state. Right: Brillouin zone of the $\mathrm{Cu}-\mathrm{O}$ plane. The dots show the half-filling Fermi surface, as well as the Brillouin zone boundary after the DDW state has formed. The circles are the Fermi surface of the DDW state at finite doping.

\section{COMPETING ORDER}

Order-parameter competition has always been a natural candidate for explaining why the superconducting transition temperature $T_{c}$ first grows and then retreats as doping is reduced. Let us consider the generic zerotemperature Ginzburg-Landau free energy

$$
F=\lambda\left(y^{2}+|z|^{2}\right)^{2}+\gamma y^{2}|z|^{2}-\alpha y^{2}-\alpha^{\prime}|z|^{2},
$$

describing the development of order parameters $y$ and $z$ in the case that low-order mixing is forbidden by symmetry. In Fig. 1b we plot the values of $y$ and $z$ that minimize $F$ for the case of $\lambda=1$ and $\gamma=-0.8$ as a function of the abstract tuning parameter $p$. The variables $\alpha$ and $\alpha^{\prime}$ are the simple linear functions of $p$ shown in Fig. 1c. One sees that $z$ develops at $p=0.3, y$ develops at $p=0.2$, and that $0.1<p<0.2$ is a coexistence region in which the growth of $y$ suppresses and eventually eliminates $z$. Thus if we imagine $z$ to be the magnitude of the order parameter for $d$-wave superconductivity and $p$ to be doping, then we can understand the onset, growth, saturation, and eventual destruction of superconductivity with reduced doping as an effect of a monotonically strengthening $d$-wave pairing interaction, as opposed to one that first strengthens and then weakens. The underdoped side of the superconducting dome is then fundamentally different from the overdoped side in that the superfluid density is suppressed there by the development of a second order parameter $y$.

\section{D-DENSITY WAVE}

Let us now consider the order parameter

$$
y=i \sum_{\mathbf{k}, s} f(\mathbf{k})<c_{\mathbf{k}+\mathbf{Q}, s}^{\dagger} c_{\mathbf{k}, s}>,
$$

where $f(\mathbf{k})=\cos \left(k_{x}\right)-\cos \left(k_{y}\right)$. If $f(\mathbf{k})$ were replaced by a function with $s$-wave symmetry, $y$ would simply be the order parameter of a charge-density wave (CDW) - hence, we call this state a $d_{x^{2}-y^{2}}$ density wave state (DDW) [3]. For the particular case of $\mathbf{Q}=(\pi, \pi)$, which we think most relevant to the cuprates, the equivalence of $\mathbf{Q}$ and $-\mathbf{Q}$ enforced by the underlying band structure requires the sum to be imaginary. Thus this state necessarily breaks parity and time-reversal symmetry (i.e. exhibits magnetism), as well as translation by one lattice spacing and rotation by $\pi / 2$. It is, however, symmetric under the combination of any two of these operations. The order parameter is equivalent to the array of bond currents illustrated in Fig. 2.

The excitation spectrum of the DDW at very low energies is generic and consists of conventional fermionic particles and holes in a band structure like that of the $d$-wave superconductor with which it competes. Introducing a mean-field ansatz [3] ( $c f$. Eq. 2) we obtain the 1-body Hamiltonian

$$
\mathcal{H}=\sum_{\mathbf{k}, \sigma} \epsilon(\mathbf{k}) c_{\mathbf{k} \sigma}^{\dagger} c_{\mathbf{k} \sigma}+\Delta(\mathbf{k}) c_{\mathbf{k} \sigma}^{\dagger} c_{\mathbf{k}+\mathbf{Q} \sigma}
$$

where $\epsilon_{\mathbf{k}}=-2 t\left[\cos \left(k_{x}\right)+\cos \left(k_{y}\right)\right]$ and $\Delta_{\mathbf{k}}=y V\left[\cos \left(k_{x}\right)-\right.$ $\left.\cos \left(k_{y}\right)\right]$, and $V$ is a coupling constant in the microscopic Hamiltonian. Microscopic Hamiltonians with short-range repulsion and superexchange are favorable for such order [5.66] but are even more favorable for an antiferromagnetic state. However, correlated hopping terms tend to tip the balance in favor of DDW order [3]. Since the ordering occurs at $\mathbf{Q}=(\pi, \pi)$, it is most favorable at half-filling or low doping. The corresponding band structure is

$$
E_{\mathbf{k}}= \pm \sqrt{\epsilon_{\mathbf{k}}^{2}+\left|\Delta_{\mathbf{k}}^{\mathrm{DDW}}\right|^{2}} .
$$

At half-filling there are gapless quasiparticles only at the nodal points $\mathbf{k}=( \pm \pi / 2, \pm \pi / 2)$. At finite doping, Fermi pockets are opened, as shown in Fig. 2. While the DDW state is semimetallic at half-filling, it is a conventional metal (with 2-d localization prevented by interlayer tunneling) with a disconnected Fermi surface at dopings other than half-filling. It is possible for the DDW to discommensurate, thereby opening a full gap, as occurs with a traditional spin density wave, but this is not automatic because the remaining Fermi surface is not nested. Some related density-wave states are discussed in the Appendix.

The excitation spectrum at high energies is not generic. There is no reason for the quasiparticle at $(\pi, 0)$ to have integrity, particularly if the system is near the continuous quantum phase transition at $p=0.2$ in Fig. 1. This is a Fermi-surface reconnection, at which the Hall conductance jumps, a van Hove singularity develops at $(\pi, 0)$, and quasiparticles scatter violently even at low energy scales [14,21,22].

\section{D-WAVE SUPERCONDUCTIVITY}

The Heisenberg exchange nominally responsible for DDW order also tends to favor $d$-wave superconductivity. 
This is the underlying reason the band structures of the two are so similar, and why the competition of these two kinds of order is natural. If we allow the superconducting bond expectation value $\left\langle c_{j \uparrow} c_{k \downarrow}\right\rangle= \pm z$ to develop, where the sign is positive on $x$ bonds and negative on $y$ bonds, the Hartree-Fock Hamiltonian becomes

$$
\mathcal{H}_{\mathrm{HF}}^{\prime}=\mathcal{H}_{\mathrm{HF}}+J \sum_{<j k>} \pm\left(z c_{k \downarrow}^{\dagger} c_{j \uparrow}^{\dagger}+z^{*} c_{j \uparrow} c_{k \downarrow}\right)
$$

and the corresponding superconducting quasiparticle dispersion relation becomes

$$
E_{\mathbf{k}}= \pm \sqrt{\left[\left(\epsilon_{\mathbf{k}}^{2}+\left|\Delta_{\mathbf{k}}^{\mathrm{DDW}}\right|^{2}\right)^{1 / 2} \pm \mu\right]^{2}+\left|\Delta_{\mathbf{k}}^{\mathrm{DSC}}\right|^{2}},
$$

where $\Delta_{\mathbf{k}}^{\mathrm{DSC}}=z J\left[\cos \left(k_{x}\right)-\cos \left(k_{y}\right)\right]$ and $\mu$ is the chemical potential. Thus not only does this kind of interaction stabilize both kinds of order, it allows the two order parameters to evolve continuously into each other without collapsing the quasiparticle gap at the zone face. This allows us to use the ground state expectation value of $\mathcal{H}$ and similar Hamiltonians as a sensible model for the energy functional $F$, i.e. one that does not throw away important low-energy excitations.

This calculation illustrates an important feature of the mixed state that the superfluid density is not fixed by sum rules on the underlying Fermi surface but is rather determined by the balance between the DDW and DSC order parameters. This is because the superfluid is primarily a condensate of Cooper pairs drawn from the gapped region near $(\pi, 0)$ rather than the residual Fermi surface near $(\pi / 2, \pi / 2)$. This effect is not difficult to understand if the DDW order parameter is small, for then the semimetallic state with Fermi points - or, away from half-filling, the conventional metallic state with a small, disconnected Fermi surface - is not significantly different from the parent metal with a full Fermi surface at the energy scales relevant to superconductivity. As the DDW order parameter becomes large, however, we have more and more the case of a powerful attractive force exciting electrons and holes virtually into the insulating part of the band structure and then binding these into superfluid. The result is a condensate fraction that falls precipitously as the DDW order parameter grows. An insulating ground state (or, in this case, nearly insulating, since there is a small disconnected Fermi surface) that becomes a superfluid without first becoming a metal is unusual in solids, but perhaps not in nature, for this is the central idea behind Higgs condensation in electroweak theory.

\section{S-WAVE COMPETITION}

The competition between DDW and DSC has a simple analogue in the $s$-wave case [23] that is particularly instructive because it is exact 24]. The Hubbard model

$$
\mathcal{H}=-t \sum_{<j k>} \sum_{\sigma} c_{j \sigma}^{\dagger} c_{k \sigma}+U \sum_{j} c_{j \uparrow}^{\dagger} c_{j \downarrow}^{\dagger} c_{j \downarrow} c_{j \uparrow}
$$

has the special property at half-filling that replacing the fermion operator on a lattice site $j, c_{j \downarrow}$, by $(-1)^{j} c_{j \downarrow}^{\dagger}$ (a unitary transformation at half-filling) reverses the sign of $U$. When $U>0$ this model has a ground state which is an ordered antiferromagnet characterized by the expectation values

$$
\left[\begin{array}{c}
<S_{j}^{x}> \\
<S_{j}^{y}> \\
<S_{j}^{z}>
\end{array}\right]=\frac{1}{2}\left[\begin{array}{c}
<c_{j \uparrow}^{\dagger} c_{j \downarrow}+c_{j \downarrow}^{\dagger} c_{j \uparrow}> \\
i<c_{j \uparrow}^{\dagger} c_{j \downarrow}-c_{j \downarrow}^{\dagger} c_{j \uparrow}> \\
<c_{j \uparrow}^{\dagger} c_{j \uparrow}-c_{j \downarrow}^{\dagger} c_{j \downarrow}>
\end{array}\right] .
$$

When $U<0$ the ground state is thus a degenerate mixture of $s$-wave superconductivity and checkerboard charge order characterized by the expectation values

$$
\left[\begin{array}{c}
<\operatorname{Re}\left(\Delta_{j}\right)> \\
<\operatorname{Im}\left(\Delta_{j}\right)> \\
<n_{j}>
\end{array}\right]=\left[\begin{array}{c}
<c_{j \uparrow}^{\dagger} c_{j \downarrow}^{\dagger}+c_{j \downarrow} c_{j \uparrow}> \\
i<c_{j \uparrow}^{\dagger} c_{j \downarrow}^{\dagger}-c_{j \downarrow} c_{j \uparrow}> \\
(-1)^{j}<c_{j \uparrow}^{\dagger} c_{j \uparrow}+c_{j \downarrow}^{\dagger} c_{j \downarrow}>
\end{array}\right]
$$

Both kinds of order occur simultaneously, are equivalent energetically, and may be rotated into each other by analogy with spin rotation of an antiferromagnet. More precisely, this system lies at a quantum phase transition between the two kinds of order and can be made to acquire one, the other, or a mixture of the two by means of an arbitrarily small perturbation, exactly the way the parameters $\gamma$ and $\alpha-\alpha^{\prime}$ in Eq. (1) break the rotational invariance of $F$.

The Hartree-Fock solution, which is only approximate, also has this symmetry. Allowing the expectation values $y=(-1)^{j}<c_{j \uparrow}^{\dagger} c_{j \uparrow}-c_{j \downarrow}^{\dagger} c_{j \downarrow}>/ 2$ and $z=<c_{j \uparrow} c_{j \downarrow}>$ we obtain for the Hartree-Fock Hamiltonian

$$
\begin{aligned}
\mathcal{H}_{\mathrm{HF}} & =-t \sum_{<j k>} \sum_{j \sigma} c_{k \sigma}^{\dagger} c_{k \sigma}+U \sum_{j}\left[( - 1 ) ^ { j } y \left(c_{j \uparrow}^{\dagger} c_{j \uparrow}\right.\right. \\
& \left.\left.-c_{j \downarrow}^{\dagger} c_{j \downarrow}\right)+\left(z c_{j \downarrow}^{\dagger} c_{j \uparrow}^{\dagger}+z^{*} c_{j \uparrow} c_{j \downarrow}\right)\right]
\end{aligned}
$$

and for the corresponding quasiparticle dispersion relation

$$
E_{\mathbf{k}}= \pm \sqrt{\left[\left(\epsilon_{\mathbf{k}}^{2}+\left|\Delta^{\mathrm{CDW}}\right|^{2}\right)^{1 / 2} \pm \mu\right]^{2}+\left|\Delta^{\mathrm{SSC}}\right|^{2}}
$$

where $\Delta^{\mathrm{CDW}}=y U$ and $\Delta^{\mathrm{SSC}}=z U$, are the charge density wave and $s$-wave superconducting gaps, respectively. This is exactly the same as Eq. (6) with $s$-wave quantities substituted for $d$-wave ones. Thus, as in the $d$-wave case, the superconducting order parameter may, at half-filling $(\mu=0)$ be rotated continuously from pure superconductivity to pure checkerboard charge order without closing the quasiparticle gap. In this case, however, the rotation 
also leaves the ground state energy invariant, and is an exact symmetry [16,24].

This calculation illustrates the important feature of charge order that it competes easily and naturally with $s$-wave superconductivity but not with $d$-wave. This is because it is an $s$-wave condensate, per Eq. (2).

\section{PSEUDOGAP}

A large number of experimental properties of the cuprates are consistent with the presence of DDW order in underdoped samples.

\section{A. Gap Evolution}

The $d$-wave superconducting gap in the electron spectral function evolves continuously with underdoping into the $d$-wave-like pseudogap without collapsing. In the top of Fig. 3 we reproduce point-contact tunneling measurements on underdoped YBCO of Renner et al. [25] showing the excessive size of the tunneling gap and its persistence above the superconducting $T_{c}$, both of which are characteristic of underdoped cuprates. Identification of this feature with the $d$-wave gap follows from its evolution out of the simpler BCS-like gap found in overdoped materials and its rough compatibility with the magnitude of $T_{c}$. However, its persistence above $T_{c}$ is not consistent with a traditional BCS gap, for this should disappear at $T_{c}$, as occurs in overdoped samples, on quite general grounds. That this gap has the correct angular dependence is shown in the middle of Fig. 3, where we reproduce angle-resolved photoemission spectra from underdoped $\mathrm{Bi}_{2} \mathrm{Sr}_{2} \mathrm{CaCu}_{2} \mathrm{O}_{8+\delta}$ at two different points in the Brillouin zone reported by Norman et al. 226]. The upper trace, taken from near the zone face at $(\pi, 0)$, shows a large gap that persists well above $T_{c}$, whereas the lower trace, taken from near the node at $(\pi / 2, \pi / 2)$, shows a smaller gap that is destroyed at $T_{c}$. This angular dependence is also seen in the bottom of Fig. 3, where we reproduce the retreat of the photoemission "leading edge" as a function of position on the weak-coupling Fermi surface reported by Harris et al. [27]. The $d$-wave-like character of the gap is clear, as is its persistence at the zone face above $T_{c}$ for even slightly underdoped samples. Thus it appears that the pseudogap and the superconducting gap have identical functional forms and evolve continuously into each other as the doping is reduced, just as expected from order-parameter rotation.

Energetic competition as the cause of this rotation is suggested by the similarity between the superconducting and pseudogap energy scales. It may be seen in the bottom of Fig. 3 that the maximum "leading-edge" gapis $30 \mathrm{meV}$ while the retreat caused by heating above $T_{c}$ is
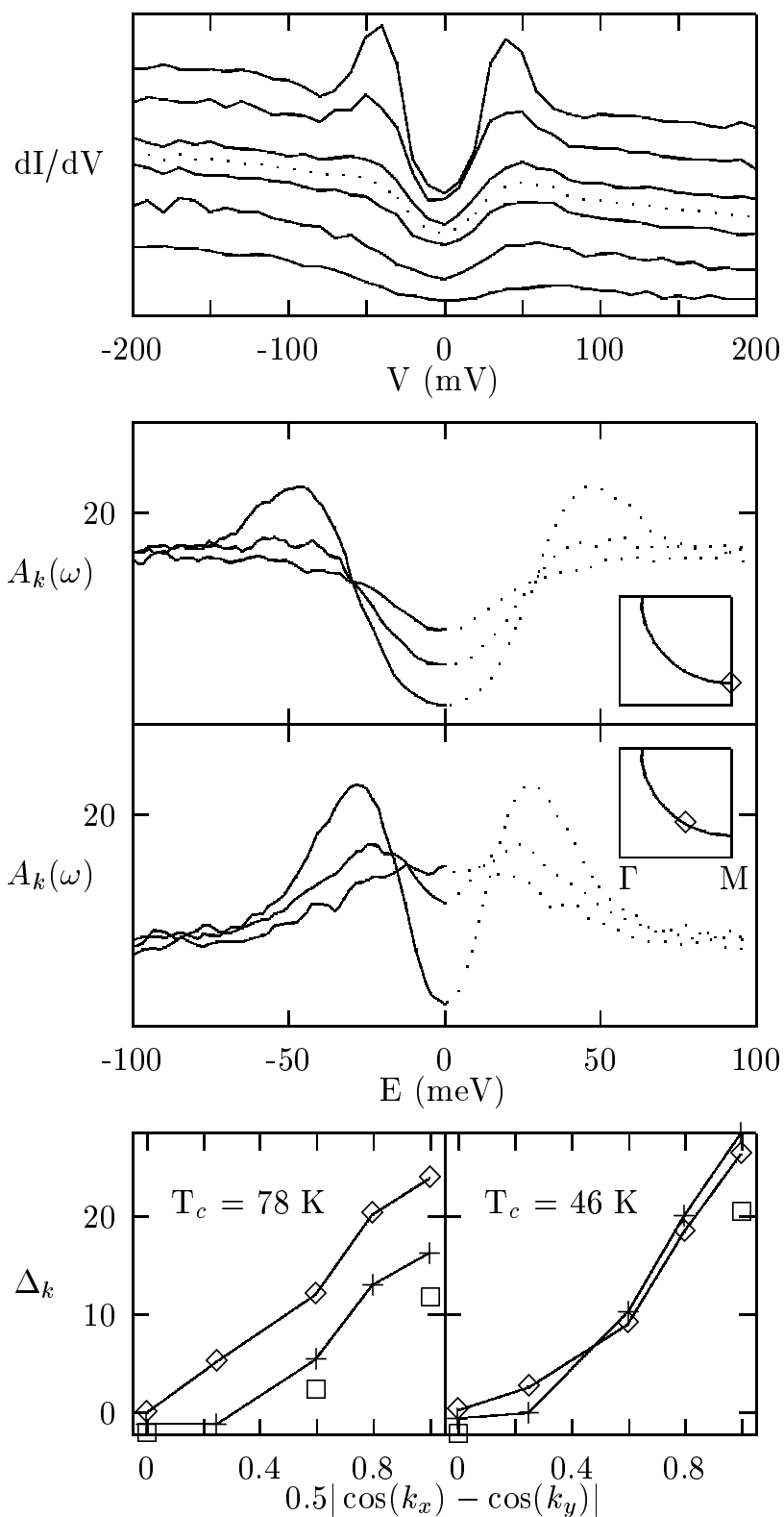

FIG. 3. Top: Point-contact tunneling spectrum of underdoped Bi2212 by Renner et al. [24] at temperatures (top to bottom) $4.2 \mathrm{~K}, 63 \mathrm{~K}, 81 \mathrm{~K}, 89 \mathrm{~K}, 109 \mathrm{~K}$, and $151 \mathrm{~K}$. The dotted line shows the spectrum at $T_{c}=85 \mathrm{~K}$. Middle: Angle-resolved photoemission from Norman et al. [25] on underdoped Bi2212 with $T_{c}=75 \mathrm{~K}$ at the two points in the Brillouin zone shown in the insets. The data were symmetrized to remove the Fermi function. The temperatures are from top to bottom: $65 \mathrm{~K}, 85 \mathrm{~K}, 110 \mathrm{~K}$. Bottom: Leading-edge gap from Harris et al. [26]. Left panel: $T_{c}=78 \mathrm{~K}$ Dy-BSCCO, at temperatures $13 \mathrm{~K}$ (diamonds), $100 \mathrm{~K}$ (crosses), $150 \mathrm{~K}$ (squares). Right panel: $T_{c}=46 \mathrm{~K}$ Dy-BSCCO; the symbols are the same as in the left panel.

between $5 \mathrm{meV}$ and $10 \mathrm{meV}$, depending on doping. The pseudogap scale $\mathrm{k}_{B} \mathrm{~T}^{*} \simeq 30 \mathrm{meV}$ is also identified in a number of other measurements [9], notably neutron 

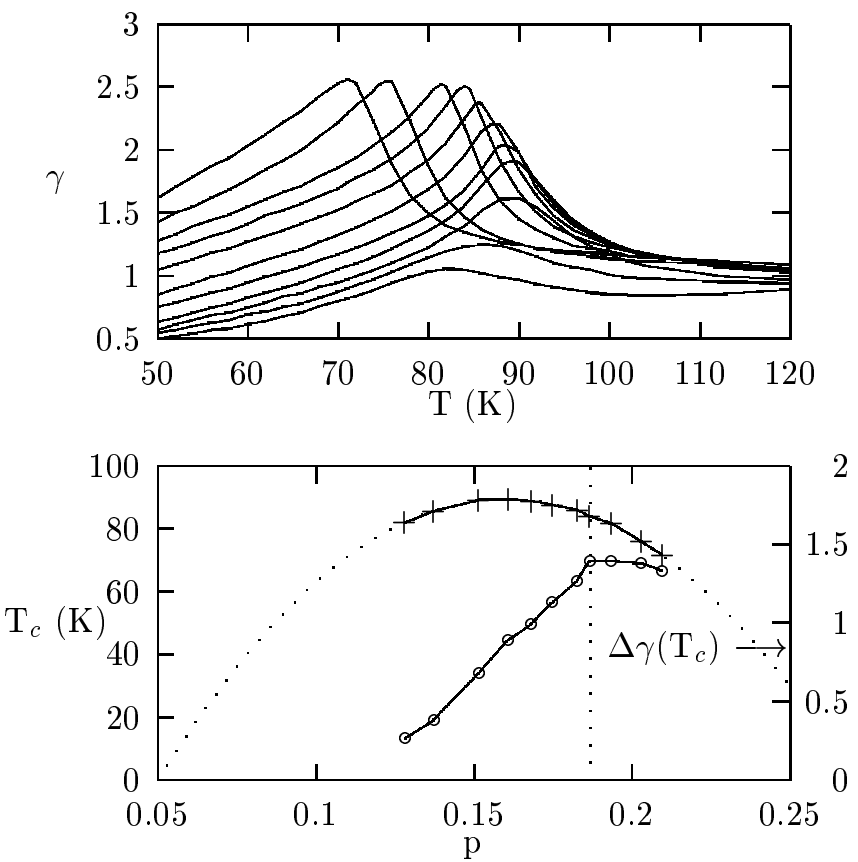

FIG. 4. Top: Specific heat $\gamma$ in $\mathrm{mJ} / \mathrm{mole}^{2}$ for various doping levels of $\mathrm{Bi}_{2.15} \mathrm{Sr}_{1.85} \mathrm{CaCu}_{2} \mathrm{O}_{8+\delta}$ of Tallon and Loram [31]. The $y$-intercepts of the curves increase with doping so that the lower curves correspond to underdoped crystals while the upper curves correspond to overdoped crystals; the fourth highest curve corresponds to optimal doping. Bottom: Specific heat jump $\left[\gamma\left(T_{c}\right)-\gamma(120 \mathrm{~K})\right]$ of above samples versus doping. The doping level is determined from $T_{c}$ and the semiempirical relation relation between this and doping $p$ in holes per $\mathrm{Cu}$ site shown in plusses.

scattering [28], NMR [29], electronic Raman scattering [30], and optical reflectivity [31].

\section{B. Superfluid Density}

Rapid collapse of superfluid density below optimal doping is seen in many experiments [32]. The zerotemperature penetration depth, for example, grows rapidly in the pseudogap regime and correlates with the suppression of $T_{c}$ with underdoping, yet saturates at overdoping in a way reminiscent of a traditional BCS superconductor 33]. In Fig. 4 we reproduce the heat capacity measurements on $\mathrm{Bi}_{2.15} \mathrm{Sr}_{1.85} \mathrm{CaCu}_{2} \mathrm{O}_{8+\delta}$ recently reported by Tallon and Loram [32]. Above a hole concentration of about $p=0.19$ per $\mathrm{Cu}$ the specific heat jump at the superconducting transition varies weakly with $\mathrm{p}$, as one would expect if the material were an ordinary metal undergoing a transition to BCS superconductivity. At $p=0.19$, however, there is an abrupt transition and a rapid decrease of this height with underdoping, as though all or part of the Fermi surface were being destroyed by the removal of holes. As a result of this, there are fewer low-energy excitations remaining to

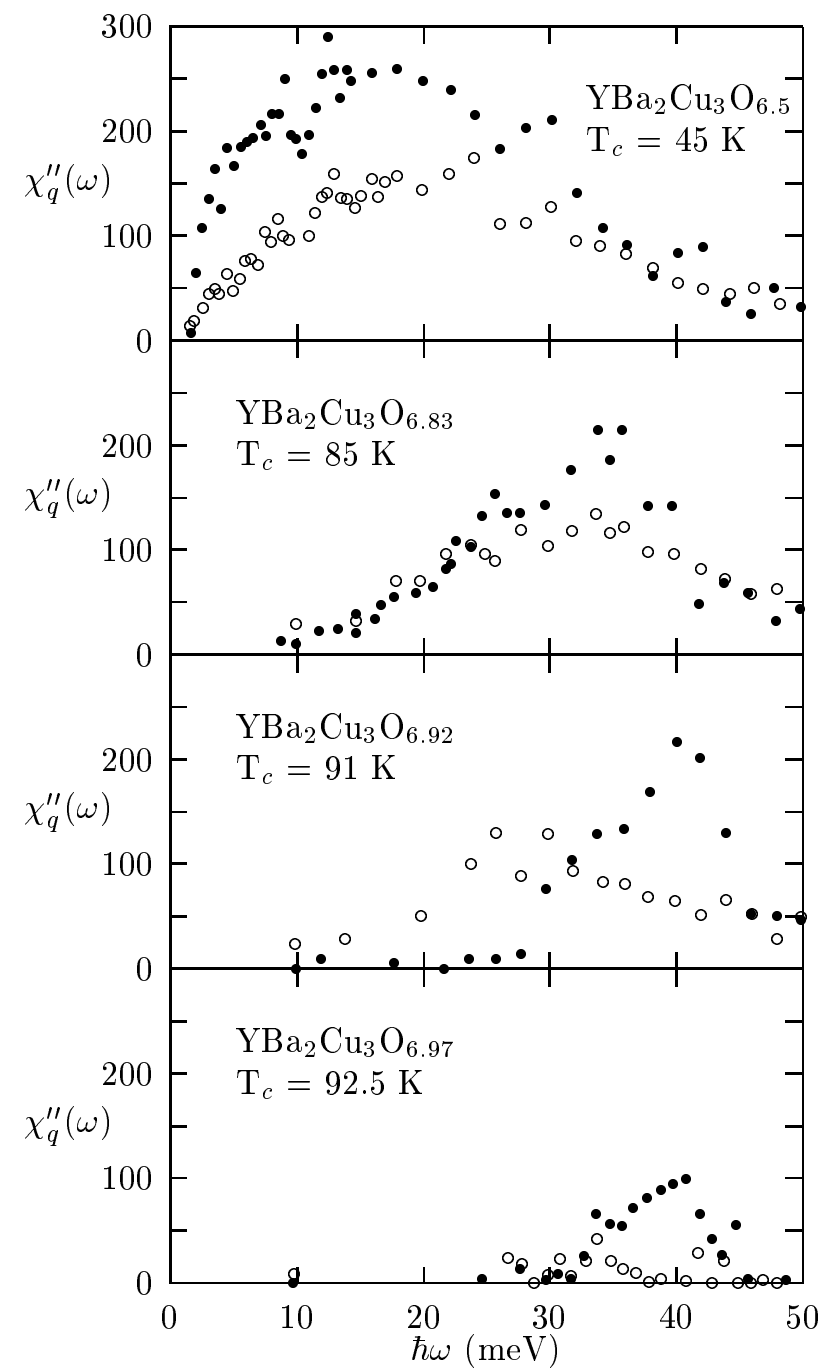

FIG. 5. Inelastic neutron scattering at $k=(\pi, \pi, \pi)$ reported by Bourges [33] for YBCO at various levels of underdoping. The closed and open circles correspond to $\mathrm{T}=5 \mathrm{~K}$ and $\mathrm{T}=100 \mathrm{~K}$, respectively. Higher-resolution experiments [30] have now shown that the shoulder on the low-energy side below $\mathrm{T}_{c}$ is actually split incommensurate peaks.

be affected by the superconducting transition. Hence, the specific heat jump at the transition, $\Delta \gamma$, is reduced. All of this behavior is compatible with Fig. 1 if the phase transition is at $p=0.2$, where the order parameter $y$ begins to develop, is associated with the onset of DDW order and the consequent continuous opening of a gap at $(\pi, 0)$ in the quasiparticle spectrum.

\section{Spin Susceptibility}

There is evidence that spin ordering - and thus presumably stripe ordering - has not taken place at optimal doping in YBCO, but only occurs at much lower doping levels. In Fig. 5 we reproduce the inelastic neutron measurements [34] for optimally-doped and underdoped 


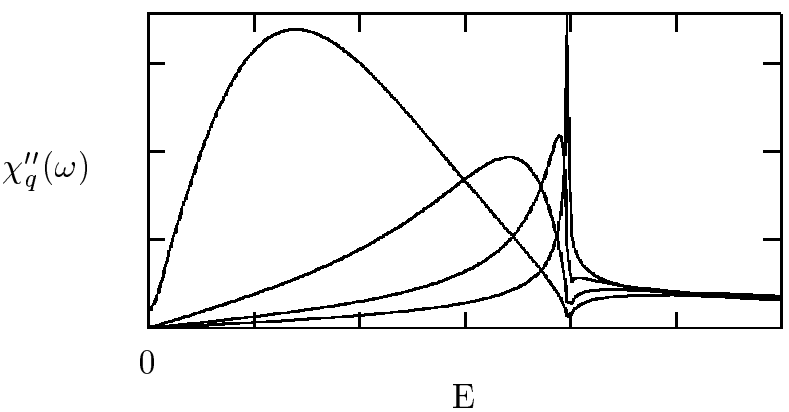

FIG. 6. $\operatorname{Im} \chi_{\mathbf{q}}(\omega)$ as defined by Eq. 13 for the case of $\mu=0, y J /(2 t+x J)=0.1$ and $U / U_{c}=0.0,0.3,0.6,0.9$, where $U_{c}$ is the critical value of $U$.

YBCO at a momentum transfer of $(\pi, \pi, \pi)$. These experiments show that the $41 \mathrm{meV}$ resonance, which disappears above $T_{c}$ and is presumably associated with the superconductivity, evolves continuously with underdoping into the magnetic fluctuation spectrum of the ordered antiferromagnet. Thus, we interpret the piling up of low-frequency spectral weight in the experiment at low doping as signaling the approach of magnetic order, and conversely of showing that magnetic order is neither present nor imminent at the onset of DDW order. The spin-fluctuation spectrum in the superconducting region remains fully gapped and has no low-energy structure of any kind. The resonance continues to be destroyed by elevated temperature, but the requisite temperature grows with underdoping even as $T_{c}$ is evolving to zero. In this way an excitation manifestly associated with the superconductivity at optimal doping transforms into an excitation irrelevant to superconductivity 35.].

This effect is simply understood as a triplet exciton [36] that vanishes at elevated temperature because the quasiparticle gap required for it to be well-defined vanishes. This is quantified in Fig. 6, where we plot the imaginary part of

$$
\chi_{\mathbf{q}}(\omega)=\frac{\chi_{\mathbf{q}}^{0}(\omega)}{1+U \chi_{\mathbf{q}}^{0}(\omega)}
$$

where

$$
\begin{aligned}
& \chi_{\mathbf{q}}^{0}(\omega)=\frac{1}{2 \pi^{2}} \int_{-\pi}^{\pi} \int_{-\pi}^{\pi} d k_{x} d k_{y} \frac{E_{\mathbf{k}}+E_{\mathbf{k}+\mathbf{q}}}{(\omega+i \eta)^{2}-\left(E_{k}+E_{\mathbf{k}+\mathbf{q}}\right)^{2}} \\
& \times\left(1-\frac{\varepsilon_{\mathbf{k}} \varepsilon_{\mathbf{k}+\mathbf{q}}+\Delta_{\mathbf{k}}^{D D W} \Delta_{\mathbf{k}+\mathbf{q}}^{D D W}+\Delta_{\mathbf{k}}^{D S C} \Delta_{\mathbf{k}+\mathbf{q}}^{D S C}}{E_{\mathbf{k}} E_{\mathbf{k}+\mathbf{q}}}\right)
\end{aligned}
$$

at $\mathbf{q}=(\pi, \pi)$ for various values of $U$. This is a crude ladder sum in which $\chi_{\mathbf{q}}^{0}(\omega)$ represents the susceptibility of the ideal BCS superconductor characterized by $E_{\mathbf{k}}$, $\Delta_{\mathbf{k}}^{\mathrm{DDW}}$, and $\Delta_{\mathbf{k}}^{\mathrm{DSC}}$ per Eq. (6), while $U$ represents a coulomb interaction added to push this system toward spin antiferromagnetism. One sees that as $U$ is increased the sharp resonance in the spectrum decreases in energy and broadens, just as occurs with decreased doping in Fig. 5. This width is due to efficient decay of the exciton into nodal quasiparticle pairs. At a slightly higher value of $U$ the continuum evolves into a divergence at $\omega=0$ associated with onset of spin order. Note that the DDW and DSC order parameters in this calculation are effectively interchangeable. Since $\Delta_{\mathbf{k}}^{\mathrm{DDW}}=-\Delta_{\mathbf{k}+\mathbf{q}}^{\mathrm{DDW}}$ and $\Delta_{\mathbf{k}}^{\mathrm{DSC}}=-\Delta_{\mathbf{k}+\mathbf{q}}^{\mathrm{DSC}}$ for $\mathbf{q}=(\pi, \pi)$, the coherence factor is unity and unchanged close to the Fermi energy whether or not both gaps, or only one of them, are present. For an $s$-wave gap the corresponding coherence factor would have been zero.

\section{High-Field Transport}

Stripes and antiferromagnetic order are naturally associated with the insulating behavior of the cuprates seen near half-filling [37. In a conventional doped band insulator, insulation is caused by impurities, which trap carriers and prevent them from moving. The system becomes a metal when it is doped sufficiently that the impurity orbitals touch. One of the most significant characteristics of the cuprates is that they continue to insulate to phenomenally high dopings, typically $5 \%$ or 1 hole for every $20 \mathrm{Cu}$ atoms. It is very difficult to understand how an insulator with an energy gap less than that of the common semiconductor GaAs should still insulate at these high dopings through impurity trapping solely. But development of antiferromagnetic order with antiphase domain walls, which then trap carriers and pin, is easy to understand, physically sensible, and supported experimentally by the simultaneous occurrence in these materials of discommensurated magnetic Bragg peaks and X-ray satellites at exactly half their momentum displacements [38]. Thus our view is that charge ordering (which would have an order parameter of the form (2), but with an $f(\mathbf{k})$ which has $s$-wave symmetry and, in all likelihood, incommensurate $\mathbf{Q}$ ) impedes conduction, rather than facilitating it [1], and moreover is characteristic of the insulating state.

The issue of coexistence of superconductivity with stripes and antiferromagnetism, and potential causative relations among them, is still highly controversial and a matter of experimental study [39]. There is, however, increasing evidence that the coexistence found in $\mathrm{La}_{2-x} \mathrm{Sr}_{x} \mathrm{CuO}_{4}: \mathrm{Nd}$ [38] is anomalous and that the cuprates with the highest values of $T_{c}$ have charge ordering only at the low-doping edge of the superconducting dome. The recent neutron scattering from $\mathrm{YBa}_{2} \mathrm{CuO}_{3} \mathrm{O}_{7-x}$ reported by Mook et al. 40 find the charge-ordering line shown in Fig. 1 and no static antiferromagnetism anywhere in the superconducting region. This is consistent with the the high-field transport experiment on $\mathrm{Bi}_{2} \mathrm{Sr}_{2-x} \mathrm{La}_{x} \mathrm{CuO}_{6+\delta}$ recently reported by Ono 


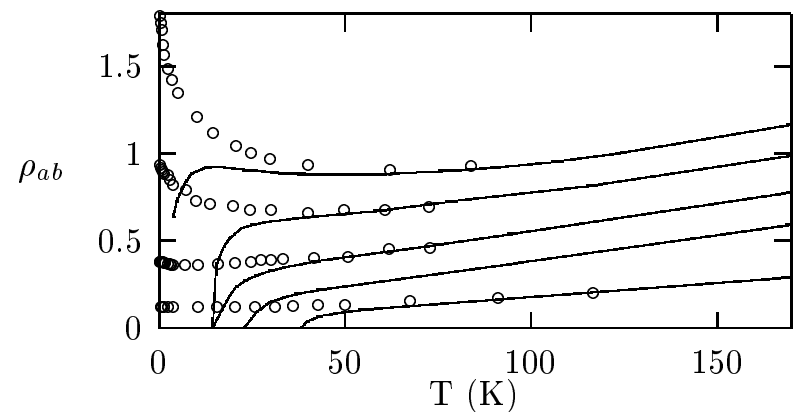

p (hole concentration per $\mathrm{Cu}$ )

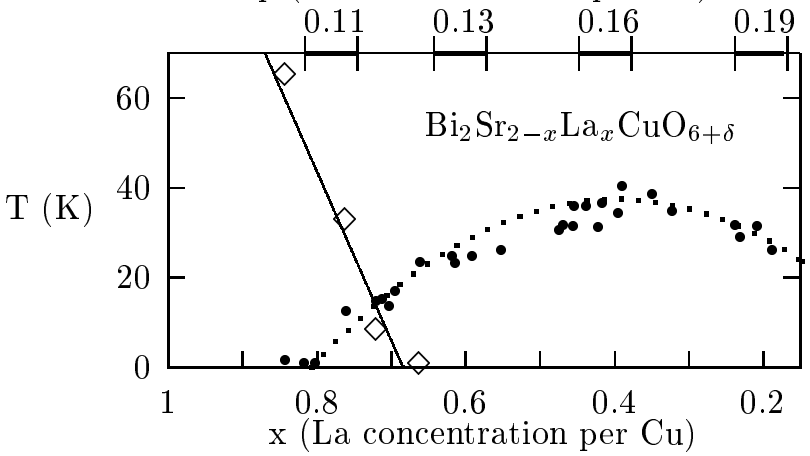

FIG. 7. High-field transport experiment of Ono et al. [40] on $\mathrm{Bi}_{2} \mathrm{Sr}_{2-x} \mathrm{La}_{x} \mathrm{CuO}_{6+\delta}$. Top: Resistivity in $\mathrm{m} \Omega$-cm versus temperature for samples with La concentrations (top to bottom) $\mathrm{x}=0.84,0.76,0.73$, and 0.39 with (circles) and without (solid lines) an applied magnetic field of $60 \mathrm{~T}$. Bottom: The temperature of the minimum value of $\rho$ (diamonds) plotted versus hole concentration. The superconducting $\mathrm{T}_{c}$ (dots) is plotted for reference.

et al. 41 reproduced in Fig. 7, which finds a metalinsulator transition at essentially the same doping as the charge ordering line of [40] when the superconductivity is crushed by a large magnetic field. The phenomenology of this transition is qualitatively similar to that observed previously in $\mathrm{La}_{2-x} \mathrm{Sr}_{x} \mathrm{CuO}_{4}$ [42] except that it occurs near the edge of the dome rather than near optimal doping. This is important, for Castellani, Di Castro, and Grilli 43] were led by this observation to propose that the strange-metal behavior of the cuprates might be quantum criticality associated with the charge-ordering transition. These more recent experiments suggest that it is instead quantum criticality associated with the development of DDW order. LSCO is unique among the high- $\mathrm{T}_{c}$ cuprates in having a low transition temperature, a strong tendency to stripe-order near $1 / 8$ doping, and an extreme sensitivity to $\mathrm{Nd}$ doping [38], all of which suggest mechanical weakness of the crystal structure.

The large-field experiment also reveals another important aspect of the cuprates, namely the lack of evidence for strange-metal behavior in the zero-temperature normal state. It may be seen in the top of Fig. 7 that the resistivities on the metallic side of the transition become constant at low temperatures and that they evolve

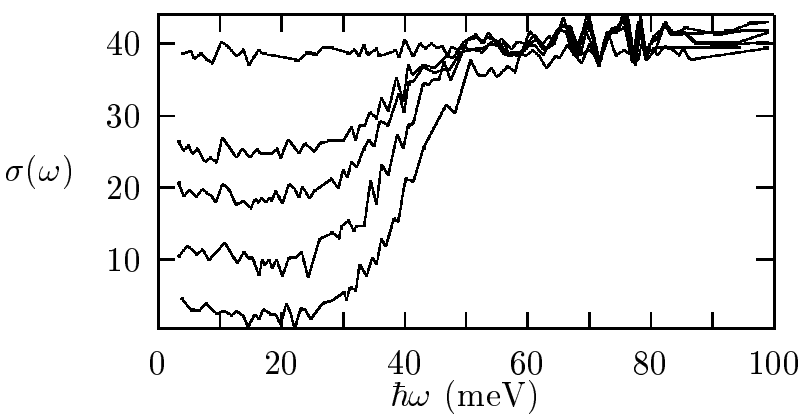

FIG. 8. $c$-axis optical conductivity of $\mathrm{YBa}_{2} \mathrm{Cu}_{3} \mathrm{O}_{6.7}$ in $\Omega^{-1} \mathrm{~cm}^{-1}$ reported by Homes et al. [43] for temperatures (top to bottom) $\mathrm{T}=250 \mathrm{~K}, 150 \mathrm{~K}, 110 \mathrm{~K}, 70 \mathrm{~K}$, and $10 \mathrm{~K}$. Large phonon contributions have been subtracted out.

continuously across the transition into the linear- $T$ resistivity characteristic of the high-temperature normal state of the cuprates. The resistivity at the transition is also about $200 \mu \Omega-\mathrm{cm}$, a typical saturation resistivity in strong-scattering metals. Both of these properties are consistent with the zero-temperature normal state being a conventional metal. They do not prove this, but they make the argument for a non-Fermi-liquid phase more difficult, as linear- $T$ resistivity is one of its key signatures.

Thus on the basis of these experiments we predict that in large magnetic fields there should be a second zerotemperature phase transition near $p=0.19$ associated with the onset of DDW order. At this transition the system should remain a conventional metal but violently change the topology of its Fermi surface. This transition should be plainly visible in all transport measurements and should be characterized by powerful critical scattering. 14, 21,22

\section{E. $c$-Axis Conductivity}

DDW formation provides a simple explanation for the perplexing semiconducting $c$-axis resistivity in many cuprates. In Fig. 8 we reproduce the optical conductivity measurements on $\mathrm{YBa}_{2} \mathrm{Cu}_{3} \mathrm{O}_{6.7}$ of Homes et al. 44. showing the steady reduction of the oscillator strength below $40 \mathrm{meV}$ beginning at a temperature far above the superconducting $\mathrm{T}_{c}$. That this reduction does not conserve the f-sum rule locally - which any mean-field theory, including that of the DDW, does - is interesting but not necessarily significant, as a mean-field description is obligated to be quantitative only at arbitrarily small energy scales. Band structure studies [45] of these materials have shown that the $c$-axis tunneling matrix element is largest at $(\pi, 0)$ and symmetry-related points - precisely at the points where the DDW gap is large. (The functional form is roughly $t_{\perp} \sim\left(\cos k_{x}-\cos k_{y}\right)^{2}$ [45.) Thus the opening of the DDW gap suppresses the $c$-axis transport because the remaining Fermi surface does not 
conduct efficiently in the c-direction due to small tunneling matrix element. The above matrix element holds for simple tetragonal materials (Hg1201, Tl1201, etc.). For body centered tetragonal materials (LSCO, Tl2201, Bi2212, etc.), the maxima of $t_{\perp}$ are shifted towards the zone center, and the effect of opening the DDW gap at $(\pi, 0)$ and symmetry-related points is weaker.

\section{ORBITAL MAGNETISM}

The distinguishing characteristic of DDW order is the magnetic field it makes. Since the possibility of spontaneous breaking of time-reversal and parity in the cuprates was first proposed in the late 1980s there have been a number of attempts to detect such fields, most of which have reported null results 46. However there has always been confusion about the size of the effects one would expect, and there have always been mysterious magnetic signals in the cuprates, including a recent report of spin antiferromagnetism coexisting with superconductivity in a sample of superoxygenated $\mathrm{La}_{2} \mathrm{CuO}_{4+y}$ with $\mathrm{y}=0.12$ and $\mathrm{T}_{c}=42 \mathrm{~K}$ 39. This fundamentally conflicts with a previous report of no magnetism in $\mathrm{La}_{1.85} \mathrm{Sr}_{0.15} \mathrm{CuO}_{4}$ [46]. We feel that the magentic experiments are so contradictory that they can at present neither rule out nor confirm the presence of DDW order.

We estimate the magnetic field at the center of a plaquette associated with DDW order to be between 1 and 30 gauss [ 4 ]. The bond currents of Fig. 2 are roughly $e \Delta^{\mathrm{DDW}} / \hbar$, where $\Delta^{\mathrm{DDW}}$ is the maximum DDW gap. If we take this to be $30 \mathrm{meV}$, we find bond currents of about $7 \mu A$. The large uncertainty in the corresponding field strength is due mainly to uncertainty in the current path. One can reasonably consider models ranging from $\mathrm{Cu}$ sites connected by $1 \AA$ "wires" to split current carried between adjacent $\mathrm{O}$ atoms.

Let us now briefly review the current experimental situation relevant to direct detection of DDW magnetism.

\section{A. Neutron Scattering}

DDW order is, in principle, visible in magnetic neutron scattering. Unfortunately the signals are quite small compared with those from ordered spins and easily overwhelmed by them. The ratio of the staggered magnetic field associated with DDW fields to that nominally produced by an ordered array of spins is

$$
\frac{B_{\mathrm{DDW}}}{B_{\mathrm{AFM}}}=\left(\frac{e \Delta^{\mathrm{DDW}}}{\hbar c r}\right)\left(\frac{m c r^{3}}{e \hbar}\right)=\frac{m r^{2}}{\hbar^{2}} \Delta^{\mathrm{DDW}}
$$

or about 0.06 , with $r=4 \AA$ taken for the bond length. Effective magnetic moments of this size are just barely detectable in the cuprates 39,47.
It is also unfortunate that the doping levels at which DDW order should be well developed lie close to the spinglass regime [48] where the system crosses over between Néel and superconducting order. The spin glass is characterized by slightly incommensurated short-range antiferromagnetism with strongly suppressed scattering intensities along one orthorhombic axis - behavior consistent with unpaired $\mathrm{Cu}$ spins pointing in the plane 47 and inconsistent with DDW magnetism. However, numerous incursions of this magnetism into the superconducting phase have been reported, in one case deeply [39], and this has always been difficult to understand from the point of view of traditional magnetic models. It implicitly raises the question of whether there might be two kinds of antiferromagnetism in the cuprates - one, due to spins, which is incompatible with superconductivity and one, due to DDW, which is fully compatible with it and associated with pseudogap formation. Spin-orbit coupling would then mix these and conceivably make them evolve into each other with increased doping.

\section{B. X-ray Scattering}

DDW order cannot be seen in X-ray scattering. The DDW order parameter is odd under time-reversal while atomic displacements are even, so there is no first-order coupling between them, and Bragg scattering through circular birefringence from the valence electrons is too weak. For a $10 \mathrm{KeV}$ X-ray of frequency $\omega$ the Bragg intensity is down by the factor $\left(\mu_{B} B^{\mathrm{DDW}} / \hbar \omega\right)^{2} \simeq 10^{-16}$ from the Bragg intensity of valence electrons - already small compared with the signal from the core electrons. The absence of an X-ray signal is a key characteristic DDW order distinguishing it experimentally from stripes.

\section{Magnetic Resonance}

The static magnetic field of ideal DDW order cannot be seen directly through $\mathrm{NMR}$ of $\mathrm{Cu}$ or $\mathrm{O}$ nuclei in ideal $\mathrm{CuO}$ planes, as these lie at centers of symmetry where the DDW magnetic field is zero. However, magnetic fluctuations associated with the onset of DDW order or a glassy state of a disorder-frustrated DDW could be seen by NMR, although it would be difficult to distinguish from antiferromagnetic spin fluctuations for the reasons stated above. Also, DDW order can in principle be seen in NMR of ions out of the Cu-O planes, such as $\mathrm{Y}, \mathrm{Ba}$, La, or Sr. It has long been established that there are unusual magnetic signals below $\mathrm{T}_{c}$ in all the cuprates, but attempts to quantify these have been plagued by the inherent model-sensitivity of NMR analysis. Tallon and Loram [49] have recently argued using the ratio of ${ }^{63} \mathrm{Cu}$ and ${ }^{17} \mathrm{O}$ spin-lattice relaxation rates analyzed with 


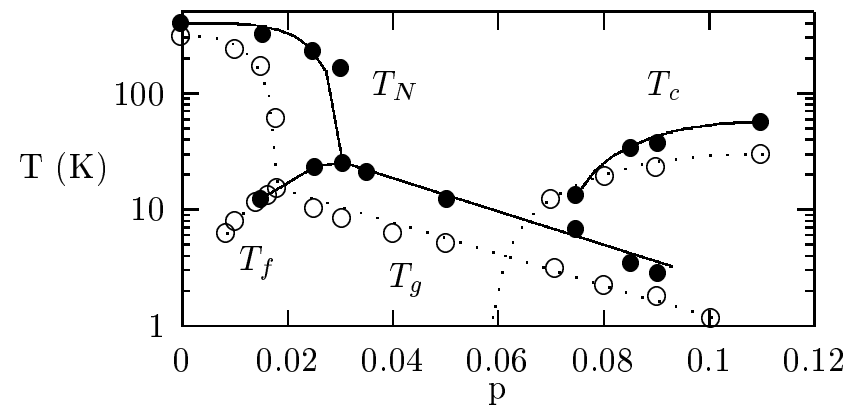

FIG. 9. Cuprate phase diagram inferred by Niedermayer et al. [47] from $\mu \mathrm{SR}$ measurements. The solid and open symbols refer to $\mathrm{Y}_{1-x} \mathrm{Ca}_{x} \mathrm{Ba}_{2} \mathrm{Cu}_{3} \mathrm{O}_{6}$ and $\mathrm{La}_{2-x} \mathrm{Sr}_{x} \mathrm{CuO}_{4}$, respectively. $T_{N}$ is the Néel temperature, $T_{c}$ is the superconducting transition temperature, $T_{f}$ is a secondary magnetic ordering that occurs on top of spin antiferromagnetism, and $T_{g}$ is the glass transition temperature. The dephasing time for the latter is approximately $0.1 \mu \mathrm{S}$.

the model of Millis, Monien, and Pines [50] that shortrange antiferromagnetic fluctuations develop in the pseudogap regime with a functional dependence on $p$ tracking roughly the value of $y$ in Fig. 1. This analysis is not persuasive evidence for DDW order.

\section{Muon Spin Resonance}

Muon spin resonance has consistently found evidence for magnetism in the superconducting state of the cuprates for dopings less than $p=0.1$. In Fig. 9 we reproduce the phase diagram of Niedermeyer et al. [51] showing boundaries of distinct magnetic behaviors observed in powders of both $\mathrm{La}_{2-x} \mathrm{Sr}_{x} \mathrm{CuO}_{4}$ and $\mathrm{Y}_{1-x} \mathrm{Ca}_{x} \mathrm{Ba}_{2} \mathrm{Cu}_{3} \mathrm{O}_{6.02}$. They report a "spin freezing" transition $\left(T_{f}\right)$ below the Néel transition, and a spinglass transition $\left(T_{g}\right)$ that cuts in to the superconducting dome. Below this transition, and at doping levels as high as $p=0.09$, the muons depolarize in about 0.1 $\mu \mathrm{S}$. In the case of LSCO, the measurements extended into the range of the $1 / 8$ anomaly at $\mathrm{p}=0.12$, beyond which the spin-glass neutron signal tends to disappear [17] and where no magnetism was found in previous $\mu \mathrm{SR}$ measurements 46]. However the fact that both cuprates behave similarly, and that the spin-glass temperature in $\mathrm{Y}_{1-x} \mathrm{Ca}_{x} \mathrm{Ba}_{2} \mathrm{Cu}_{3} \mathrm{O}_{6}$ is substantially higher, suggests that this behavior is characteristic of the cuprates as a class. Also, the way the spin-glass line ends has always been confusing.

In a recent paper Panagopoulos et al. [52 have reported anomalous long-time magnetic fluctuations at temperatures just above the glass transition in $\mathrm{La}_{2-x} \mathrm{Sr}_{x} \mathrm{CuO}_{4}$ powders. The scale of these is comparable to $\mathrm{T}_{c}$ and has a functional dependence with doping identical to that of the parameter $y$ in Fig. 1 - i.e. decreasing with doping and vanishing at $p=0.17$. Thus they argue that the spin-glass line actually ends here, not at $1 / 8$. The observation of the same effect in a different cuprate, which seems likely in light of Fig. 9, would suggest an intrinsic magnetic signal developing at the onset of DDW order.

\section{DISORDER AND CROSSOVER}

The muon phenomenology suggests an answer to a question plaguing the idea of competing order in the cuprates, namely why there is no evidence for a genuine phase transition at the pseudogap temperature $T^{*}$, the alleged phase boundary for onset of DDW, and also why previous searches for magnetism at optimal doping have found sample-dependent or null results. It is simply that the DDW order is corrupted by disorder and transformed into the spin-glass transition at the lower temperature $T_{g}$ [53. In very dirty samples it is lowered so much as to be effectively destroyed.

There has been controversy over how much intrinsic disorder cuprates possess since they were discovered. The essence of the problem is that the most sensitive tests of disorder - transport and the degradation of superconductivity - are corrupted by the non-Fermi-liquid behavior of the normal state [20] evidenced by resistivities which exceed the Ioffe-Regel limit of $100 \mu \Omega$-cm at $\mathrm{T}_{c}$ (cf. Fig. 7 ) and increase with temperature from there. However, using criteria less dependent on the theory of metals, the case for chronic disorder is easier to make: All cuprates lose oxygen easily in arbitrary amounts. All of them have spin-glass phases at low dopings [52]. All of them have magnetic scattering in the superconducting regime that is sample-dependent, difficult to reproduce, and difficult to quantify [40]. All of them have anomalous widths in $\mathrm{Cu}$ and $\mathrm{O}$ NMR and NQR [54]. Thus our view is that all cuprates made thus far have been significantly disordered, even ones showing evidence to the contrary such as narrow superconducting transition widths. This view is supported by new scanning tunneling microscope experiments on atomically perfect cleaves of optimally-doped BSCCO that find inhomogeneities in the tunneling density of states on the scale of $20 \AA$ [55].

We note that the disorder need not occur within the $\mathrm{CuO}_{2}$ planes to have a strong effect on the electronic properties. The non-superconducting cuprates which were studied in the late 1980s differ from the superconducting ones only in the elements that sit between planes. Substituting $\mathrm{Hg}$ between the planes raises $\mathrm{T}_{c}$ substantially. Substituting Nd causes stripes 38.

The DDW transition is in the same universality class as the random-bond Ising model. The DDW order parameter breaks translational and rotational symmetries, and thus couples to disorder as in a model with a random uniaxial anisotropy. From the Imry-Ma argument 53 as adapted to the random anisotropy case [56], we know that this symmetry-breaking transition will be spoiled 
by the random distribution of impurities. Thus, in the presence of disorder, time-reversal is the only true symmetry that can be spontaneously broken by the DDW state. The universality class is then that of a $Z_{2}$ symmetry preserved by the impurities.

The phase diagram of the random-bond Ising model depends critically on the disorder strength. Weak disorder is an irrelevant perturbation and can be ignored at the finite-temperature transition to a state with broken time reversal symmetry. Such a state has a nonvanishing expectation value for the staggered orbital magnetization (thereby breaking the disorder-averaged translational symmetry). If the disorder is strong, on the other hand, and the interlayer coupling is finite, then there can be a finite-temperature transition in the same universality class as the three-dimensional (3D) Ising spin-glass transition. Due to the weakness of the magnetic coupling between the planes, the spin-glass transition temperature estimated from the two-dimensional spin-glass susceptibility [57], $\chi_{s g}^{2 D} \sim T^{-\gamma}, \gamma \approx 5.3$, is small. Such a transition would not be possible if we could neglect the coupling between the planes, as the lower critical dimension of the Ising spin glass is known to be greater than two.

In contrast to this, the finite temperature transition to DSC remains sharp in the presence of disorder - although $T_{c}$ may be degraded. This is because disorder does not couple linearly to the order parameter as a random field, and because a superconducting transition in two dimensions is possible. The ultimate 3-dimensional transition driven by the coupling between the layers is robust. This can be further understood by invoking the Harris criterion [53] assuming that the transition is in the $3 D$-XY universality class. The criterion states that weak disorder is an irrelevant perturbation to the pure system if the specific heat exponent is negative, which is indeed the case for the $3 D$-XY model. When the disorder is so strong that $k_{F} l \sim 1$, where $k_{F}$ is the Fermi wave vector and $l$ is the mean free path, a superconductor-insulator transition will take place, and the Harris criterion will no longer apply.

The potential presence in this system of a disordersensitive, purely electronic, phase transition involving a Fermi-surface reconnection raises the disturbing possibility that many experiments in this field may be measuring corrupted critical properties of the DDW transition rather than the properties of new states of matter. The notorious non-Fermi-liquid behavior of the normal state, for example, appears to evolve at the lowest temperatures and in a strong magnetic field into behavior of a traditional metal. A possible explanation of this is that the high-temperature behavior is characteristic of a quantum critical [58] region associated with a nearby critical point.

\section{SUMMARY}

In summary we find that most of the strange behavior of the cuprate superconductors is consistent evidence for the simultaneous occurrence of $d$-wave superconductivity and bond antiferromagnetism. On the basis of this we predict that the spin-glass transition temperature observed in muon spin resonance will climb to the pseudogap temperature $T^{*}$ as the sample quality improves, that the onset of this effect with doping coincides perfectly with the loss of superfluid density at $p=0.19$, and that this transition will be found to be a metal-metal transition involving a Fermi surface reconnection, not a transition to stripe order $11,13,37,38$, when magnetic fields sufficiently intense are available to crush the superconductivity at optimal doping.

\section{ACKNOWLEDGEMENTS}

RBL wishes to thank Z.-X. Shen, D. Pines, S.-C. Zhang, and G. Aeppli for numerous helpful discussions. RBL, SC, and DM wish to thank the Institute for Complex Adaptive Matter at Los Alamos, where key ideas for this work were conceived. RBL was supported by the National Science Foundation under Grant No. DMR-9813899 and by NEDO. SC was supported by the National Science Foundation under Grant No. DMR9971138 and, in part, by funds provided by the University of California for the conduct of discretionary research by Los Alamos Natonal Laboratory, under the auspices of the Department of Energy. CN was supported by the National Science Foundation under Grant No. DMR9983544 and the Alfred P. Sloan Foundation. DM was supported by the Department of Energy at Los Alamos National Laboratory.

\section{APPENDIX: RELATED DENSITY WAVES}

There are two related unconventional density wave order parameters potentially relevant to the cuprates [3]. The first is that the frustration of the singlet DDW order parameter can lead to incommensurate ordering, in analogy with the Ferrell-Fulde-Larkin-Ovchinnikov [59] state in superconductivity as nesting is destroyed. As in the superconducting case, this will take place for sufficiently strong frustration and at sufficiently low temperatures. Note that in this case the order parameter is allowed to couple with lattice displacements and can therefore be seen in X-ray scattering. When the order parameter is incommensurate, it will no longer have pure $d_{x^{2}-y^{2}}$ symmetry, but will mix in $p$-wave terms. For $\mathbf{Q}=(\pi / a, \pi / a)+\mathbf{q}$ with $|\mathbf{q}|$ small, the order parameter will take the form of 
equation (2), with $f(\mathbf{k})=\cos \left(k_{x}\right)-\cos \left(k_{y}\right)$ replaced by [3]

$$
\begin{aligned}
& f(\mathbf{k})=\left(1+\frac{i}{2} q_{x} a\right)\left[\cos \left(k_{x} a\right)-\cos \left(k_{y} a\right)\right] \\
& -\frac{1}{2} q_{x} a \sin \left(k_{x} a\right)-\frac{1}{2} q_{y} a \sin \left(k_{y} a\right) .
\end{aligned}
$$

The second interesting order parameter is the triplet version of the DDW [3]. This is defined by

$$
\vec{y}=\sum_{k} f(\mathbf{k}) \sum_{s s^{\prime}} \vec{\sigma}_{s s^{\prime}}\left\langle c_{\mathbf{k}+\mathbf{Q}, s}^{\dagger} c_{\mathbf{k}, s^{\prime}}>,\right.
$$

where $\vec{\sigma}$ is a Pauli spin matrix. If $f(\mathbf{k})$ were chosen to be a function of $s$-wave symmetry, this would be a conventional spin-density wave. The order in this case is chracterized by broken time-reversal, translational, and rotational invariances. The combination of any two of time-reversal, a translation by one lattice spacing, or a rotation by $\pi / 2$ is preserved, however. In addition, spinrotational symmetry is also broken, which leads to gapless spin-1 excitations. The triplet DDW corresponds to an alternating pattern of spin currents analogous to charge currents of Fig. 2. Presently, the phenomenology of high temperature does not seem to be consistent with the choice of the triplet DDW as the competing order parameter.

[1] B. I. Halperin and T. M. Rice, Solid State Phys. 21, 116 (1968).

[2] H. J. Schulz, Phys. Rev. B 39, 2940 (1989).

[3] C. Nayak and F. Wilczek, cond-mat/9510132 (unpublished); C. Nayak, cond-mat/0001303 and condmat/0001428.

[4] T. Hsu, J. B. Marston, and I. Affleck, Phys. Rev. B 43, 2866 (1991).

[5] J. B. Marston and I. Affleck, Phys. Rev. B 43, 11538 (1989).

[6] G. Kotliar, Phys. Rev. B 37, 3664 (1988).

[7] D. A. Ivanov, P. A. Lee, and X.-G. Wen, Phys. Rev. Lett. 84, 3958 (2000).

[8] X.-G. Wen and P. A. Lee, Phys. Rev. Lett. 76, 503 (1996).

[9] T. Timusk and B. Statt, Rep. Prog. Phys. 62, 61 (1999).

[10] C. M. Varma, Phys. Rev. Lett. 83, 3538 (1999).

[11] V. J. Emery, S. A. Kivelson, and O. Zachar, Phys. Rev. B 56, 6120 (1997).

[12] S. Kivelson, E. Fradkin, and V. Emery, Nature 393, 550 (1998).

[13] S. Caprara et al., J. de Phys. IV (Proceedings) 9, 329 (1999).

[14] J. Schmalian, D. Pines, and B. Stojkovic, Phys. Rev. Lett. 80, 3839 (1998).
[15] P.W. Anderson, Science 288, 480 (2000), and references therein.

[16] S.C. Zhang, Science 275, 1089 (1997).

[17] L. Balents, M.P.A. Fisher, C. Nayak, Int. J. Mod. Phys. B 12, 1033 (1998).

[18] M. Randeria, in Proceedings of the tenth International School of Physics "Enrico Fermi", Varenna 1997, edited by G. Iadonisi and J. R. Schrieffer (IOS Press, Amsterdam, 1998).

[19] Q. Chen et al., Phys. Rev. Lett. 81, 4708 (1998)

[20] C. M. Varma, Phys. Rev. Lett. 63, 1996 (1989); E. Abrahams and C. M. Varma, cond-mat/0006055.

[21] I. Dzyaloshinskii, J. Phys. I France 6, 119 (1996).

[22] A. V. Chubukov and D. K. Morr, Phys. Rep. 288, 355 (1997).

[23] G. Bilbro and W. L. McMillan, Phys. Rev. B 14, 1887 (1976).

[24] E. Fradkin, Field theories of Condensed Matter Systems (Addison-Wesley, Redwood City, 1991); C. N. Yang and S. C. Zhang, Mod. Phys. Lett. B 4, 759 (1990).

[25] Ch. Renner et al., Phys. Rev. Lett. 80, 149 (1998); see also J. L. Tallon and G. V. M. Williams, ibid., 82, 3725 (1999); Ch. Renner et al., ibid., 3726 (1999).

[26] M. R. Norman et al., Nature 392, 157 (1998).

[27] J. M. Harris et al., Phys. Rev. B 54, R15665 (1996).

[28] P. Dai et al., Science 284, 1344 (1999).

[29] R. E. Walstedt, R. F. Bell, and D. B. Mitzi, Phys. Rev. B 44, 7760 (1991).

[30] G. Blumberg, M. V. Klein, K. Kadowaki, C. Kendziora, Science 278, 1427 (1997); F. Slakeyet al., Phys. Rev. B 42, 2563 (1990).

[31] A. V. Puchkov, D. N. Basov, and T. Timusk, J. Phys. Cond. Matt. 8, 10049 (1996); C. C. Homes et al., Phys. Rev. Lett. 71, 1645 (1993).

[32] J. L. Tallon and J. W. Loram, "The Doping Dependence of $\mathrm{T}^{*}$ - What is the Real High- $\mathrm{T}_{c}$ Phase Diagram?", submitted to Physica C cond-mat/0005063; J. L. Tallon et al., Phys. Stat. Sol. B 215, 531 (1999); J. W. Loram et al., J. Phys. Chem. Solids 59, 2091 (1998).

[33] Y. J. Uemura et al., Phys. Rev. Lett, 62, 2317(1989).

[34] P. Bourges, in The Gap Symmetry and Fluctuations in High-Temperature Superconductors, ed. by J. Bok, G. Deutscher, D. Pavuna and S. A. Wolf (Plenum Press, 1998) cond-mat/9901333; H. F. Fong et al., Phys. Rev. B 61, 14773 (2000).

[35] S. Chakravarty and H.-Y. Kee, Phys. Rev. B 61, 14821(2000) .

[36] A. Abanov and A. V. Chubukov, Phys. Rev. Lett. 83, 1652 (1999); H. Westfahl Jr. and D. K. Morr, Phys. Rev. B 62, 5891 (2000).

[37] J. Zaanen, Physica C 317 - 318, 217 (1999), and references therein.

[38] J. M Tranquada et al., Nature 375, 561 (1995); ibid., Phys. Rev. B 54, 7489 (1996).

[39] Y. Lee et al., Phys. Rev. B 60, 3643 (1999).

[40] H. A. Mook, P. Dai, F. Dogan, J. A. Fernandez-Baca, and R. D Hunt, "Observation of a Charge-Ordered State in $\mathrm{YBa}_{2} \mathrm{Cu}_{3} \mathrm{O}_{7-x}$ Superconductors", preprint.

[41] S. Ono et al., Phys. Rev. Lett. 85, 638 (2000).

[42] G. S. Boebinger et al., Phys. Rev. Lett. 77, 5417 (1996).

[43] C. Castellani, C. Di Castro, and M. Grilli, Phys. Rev. 
Lett. 75, 4650 (1995); ibid., Z. Phys. B 103, 137 (1997).

[44] C. C. Homes et al., Phys. Rev. Lett. 71, 1645 (1993).

[45] S. Chakravarty et al., Science 261, 337 (1993); O. K. Andersen et al., J. Phys. Chem. Solids 56, 1573 (1995).

[46] R. F. Kiefl et al., Phys. Rev. Lett. 63, 2136 (1989).

[47] S. Wakimoto et al., Phys. Rev. B 61, 3699 (2000); M. Matsuda et al., ibid., 4326 (2000).

[48] F. C. Chou et al., Phys. Rev. Lett. 75, 2204 (1995).

[49] J. L. Tallon and J. W. Loram, Proc. of $\mathrm{M}^{2} \mathrm{~S}$ Conference, Houston, Feb 2000 (to be published in Physica C) condmat/0005063.

[50] A. J. Millis, H. Monien, and D. Pines, Phys. Rev. B 42, 167 (1990).

[51] Ch. Niedermeyer et al., Phys. Rev. Lett. 80, 3843 (1998).

[52] C. Panagopoulos et al., cond-mat/0007158.

[53] J. Cardy, Scaling and Renormalization in Statistical Physics (Cambridge University Press, Cambridge, 1996); K. H. Fischer and J. A. Hertz, Spin Glasses (ibid., 1991).

[54] P. C. Hammel et al., Phys. Rev. B 57, R712 (1998); J. Haase et al., J. Supercond. 13, 723 (2000)

[55] J. C. Davis, private communication.

[56] R. A. Pelcovits, E. Pytte, J. Rudnick, Phys. Rev. Lett. 40, 476 (1978).

[57] R. R. P. Singh and S. Chakravarty, Phys. Rev. Lett. 57, 245 (1986).

[58] S. Sachdev, Science 288, 475 (2000), and references therein.

[59] R. Ferrell and P. A. Fulde, Phys. Rev. 135, A550 (1964); A. Larkin and Yu. N. Ovchinnikov, Sov. Phys. JETP 20, 762 (1965). 